https://dx.doi.org/10.4314/rjeste.v2i1.8

\title{
Mathematical Modelling of the effects of peer-educators' campaign on the dynamics of HIV/AIDS in Rwanda
}

\author{
Wellars Banzi ${ }^{1, *}$, Titus K.Rotich ${ }^{2}$, Jean Marie Ntaganda ${ }^{1}$ \\ ${ }^{1}$ University of Rwanda, College of Science and Technology, School of Science, Department of Mathematics. \\ ${ }^{2}$ MOI University. School of Education. \\ * Corresponding address: Wellars Banzi, webanzi@gmail.com
}

\begin{abstract}
In this paper, we analyse the effects of peer-educator's campaign on the dynamics of HIV. We present a sex-structured model for heterosexual transmission of HIV/AIDS in a community. The model is formulated using integro-differential equations, which help to account for a time delay due to incubation period of infective before developing AIDS. The sex-structured HIV/AIDS model divides the population into two subpopulations, namely; females and males. Both disease Free equilibrium and the endemic equilibrium points for the model are determined and their stability are examined. The model is extended to assess the effect of peer- educational campaigns in slowing or eradicating the epidemic. The exposure risk of infection after each intervention is obtained. Basic reproductive numbers for these models are computed and compared to assess the effectiveness of each intervention in a community. The models are numerically analyzed to assess the effectiveness of the treatment free measure, namely; peer educational campaign on the transmission dynamics of HIV/AIDS using demographic and epidemiological parameters of Rwanda. The study demonstrates the use of sex-structured HIV/AIDS models in assessing the effectiveness of educational campaigns as a preventive strategy in a heterosexually active population
\end{abstract}

\section{Mathematics Subject classifications (MSC 2010): 34D20, 34K60, 92D25, 34K25, 34K28}

Key words: Population dynamics, Stability, Basic reproductive numbers, Equilibrium

\section{Introduction}

One of the most serious global public health menaces is the human immunodeficiency virus (HIV) which emerged since in the 1980s. It is associated with the syndrome of opportunistic infections which lead to the late stage HIV disease, known as the acquired immunodeficiency syndrome (AIDS). HIV/AIDS dynamics provides a large number of new problems to mathematicians, biologists, physicians and epidemiologists because it has many features different from traditional infectious diseases and its study has stimulated the recent development of mathematical epidemiology. This virus has been killing people for more than 3 decades and will continue doing so if no nothing is made towards better condition. According to UNAIDS, the estimated number of people living with HIV is 34 million in the world with 2.5 million newly infected and 1.7 million AIDS deaths occurring. Of these, 23.5 million live in Sub-Saharan Africa [15]. Since HIV/AIDS epidemic first became visible, research on how to prevent transmission has been conducted. Mathematical models enable us to make predictions about the qualitative behaviour of disease 
outbreaks and evaluation of the impact of prevention or intervention strategies. Various mathematical models have been proposed to describe the population dynamics of HIV/AIDS, see for example [19]. These models tended to focus on the theoretical study of the HIV/AIDS. Incorporation of interventions into these models has attracted significant attention in recent years [1]. Efforts to respond to the disease surfaced early in Uganda. One prevention effort in Uganda was ABC, (Abstinence, Be faithful and Condom use). Abstinence has not borne much fruits especially among the young, unmarried people and married couples have failed to be faithful to their often-monogamous wives thus making the use of condom the only option essential.

Numerous experimental and classical studies have been conducted to ascertain the effectiveness of condom use as a tool for controlling the spread of HIV. The overall consensus of estimated condom effectiveness is between $60 \%-90 \%$ with a mean of $87 \%$ (see for instance [11], [16])

The most conclusive evidence of condom effectiveness in reducing HIV/AIDS transmission has come from studies of serodiscordant couples, and another evidence is the Thailand $100 \%$ condom policy, which required commercial sex workers and their clients to use condoms every act of intercourse, which reduced sexually transmitted infections (STIs) from 410,406 in 1987 to 29,362 in 1994 with increase in condom use from 14\% to 94\% [14], [18].

Different studies presenting models have been done to assess the effects of condom use in controlling HIV/AIDS in a community (See for instance [5], [8], [9], [10], [11], [12] just to mention a few). Analysis of the dynamics of a two-group deterministic model for assessing the impact of condom use on the sexual transmission of HIV/AIDS within homosexual population was done by Greenhalgh et al. [5]. Their study show that although condom use reduce the reproduction number $\left(R_{0}\right)$ to values less than one, it does not guarantee eradication of the disease, controlling the initial sizes of the susceptible and infected populations (for $R_{0}<1$ ) can lead to eradication of the disease. Moghadas S.M et al. [11] constructed and analyzed a deterministic model for assessing the effect of condom use based on efficacy and compliance in controlling HIV/AIDS in a community. Their study focused on determining the minimum condom efficacy and compliance rates needed for community - wide eradication of HIV/AIDS and in their analysis, they showed the product of efficacy and compliance, preventability $(\rho)$ has negative effect on the epidemic as increasing $\rho$, decreases the level of epidemicity. Further they showed that the threshold preventability $\left(\rho_{c}\right)$ increases with increasing average number of HIV- infected patterns of susceptible individuals especially in the AIDS stage.

In this paper, we construct a sex-structured HIV/AIDS model using differential equations which is shown to be equivalent to delay differential equations, to analyze the effect of peer-educator's campaign which in this context is used to mean any type of educational campaign which attempts to reduce high risk behaviours.

The mathematical model subdivides the human population under consideration into three classes that are, susceptible $S_{i}(t)$, Infected $I_{i}(t)$ and AIDS cases $A_{i}(t)$, where $i=f, m$, denotes female or male populations respectively. Each class is further subdivided into two compartments, high risk and low risk which will be denoted by the subscripts 1 and 2 respectively. The mathematical model only considers heterosexual transmission of HIV. This is because HIV transmission vertically and through intravenous methods can be totally controlled using Prevention of Mother to-Child Transmission (PMCT) and blood screening respectively. The few cases which arise from these methods are insignificant in the HIV/AIDS dynamics thus neglected in our mathematical model. Sex structured models have been studied by several authors [2], [3], [6], [13]. Hirsch in [6], 
[7] studied sex structured model for HIV treatment and behavior change, in which the sex structured model is reduced to simpler one -sex formulation using a conservation of contacts under assumption that each heterosexual contact involves only one male and one female. Related work can be found in [13]. They incorporated a parameter which modeled the number of sexual partners interacting with each infective. They modeled HIV/AIDS dynamics incorporating discrete time delay to capture the long incubation period. In their work, they did not consider transmission by AIDS cases. To the best of our knowledge, no work has been done on developing mathematical models for assessing the effect of peer-educator's campaign in controlling HIV/AIDS in the context of sex-structured incorporating discrete time delay due to the long incubation period of the disease, especially with the use of anti-retroviral drugs ( ARVs) which prolongs the survival time of HIV- infected individual, but not lead to viral eradication within infected individuals hence do not cure and over time causes the pool of potential transmitters to grow. In this study, we will also incorporate transmission by AIDS patients who are currently not easily identified because of the use of ARVs. Unlike most of the developed HIV/AIDS models, we consider male and female populations interaction, and use a constant to model the number of sexual exposures instead of number of sexual patterns, which is a more realistic approach in the modeling of HIV/AIDS preventive and therapeutic strategies because:

(a) Some intervention strategies depend on sex such as vaginal microbicides and condoms. In case of condoms, there are male and female condoms,

(b) HIV transmission from male to female and female to male are different, the probability of transmission from male to female is high due to the large mucosal structure of the vagina and the high concentration of the virus in semen [13],

(c) Number of sexual exposures is a better measure because it takes into account sexual exposures with non-sexual patterns, e.g. having forced sex through rape, and the fact that the probability of infection depends on the number of sexual acts and not only the number of sexual patterns,

(d) Number of sexual exposures for males and females are usually different in a community. We begin by developing a general sex-structured population with no peer-education. Thresholds and equilibrium are determined and stabilities examined. We extend the model to incorporate peer education campaign. The effect of $R_{0}$ is assessed when peer education is introduced. The model is numerically analysed to assess the effectiveness of using public health education in a community using demographic and epidemiological parameters of Rwanda.

This paper is organized as follows. The section 2 deals with setting on a sex-structured HIV/AIDS mathematical model. The positivity of solutions and the stability of disease-free equilibrium and endemic equilibrium are presented in this section. The peer- educators' campaign mathematical model equations are presented in the section 3 . This section focuses on the stability of equilibrium points and basic reproduction number for this model. The situation in Rwanda related mathematical model of previous section is trailed in section 4 where the numerical simulations are done. The summary and concluding remarks are presented in section 5 . 


\section{Mathematical Model Formulation}

We begin by formulating and studying a sex-structured HIV/AIDS model with any other intervention. The model divides the population into classes or compartments which are:

(a) Susceptible $S_{i}(t)$, containing individuals in sex group $i$ who have had no contact with the virus,

(b) Infected $I_{i}(t)$, individuals in sex group $i$ who are infected with the virus but have not yet developed AIDS symptoms

(c) AIDS cases $A_{i}(t)$, individuals who have developed fully symptomatic AIDs and exhibit specific clinical features

The subscript $i=f, m$ denotes female and male respectively.

The variables and parameters are defined as follows:

(i) $S_{i}(t), I_{i}(t), A_{i}(t), i=f, m$ Susceptible, Infected and AIDS cases

(ii) $\Lambda$ is the constant sexual maturity rate

(iii) $\mu$ is the natural death rate

(iv) $v$ is the accelerated death rate due to HIV infection or AIDS case

(v) $\tau$ is the constant incubation period

(vi) $\beta_{i}, i=f, m$, is the per exposure risk of infecting the other pattern when no protection of any kind is used

(vii) $c_{i}, i=f, m$, denotes the constant number of sexual exposures per year

(viii) $m$ is the per capita emigration rate

(ix) $\rho$ is the proportion of children who will mature to be females

(x) $(1-\rho)$ the complimentary proportion of children who will mature to be males

(xi) $\eta$ is the sexual contact rate of a susceptible with an AIDS case

(xii) $\quad N_{i}(t)=S_{i}(t)+I_{i}(t)+A_{i}(t)$ where $i=f, m$ proportion of sexual interacting males and females

The parameters $\eta$ in (xi) models the relative infectiousness of individuals infected with HIV and showing clinical symptoms of AIDS. This is because individuals in AIDS stage of infection are more infectious than individuals who have not progressed to AIDS stage. This is so because people in AIDS stage have a higher viral load compared with their counterparts and there is a positive correlation between viral load and infectiousness.

In order to have explicit dynamical relations we make the following model assumptions:

(a) The model assumes that vertical and intravenous transmission of HIV is minimal and can be ignored and only HIV transmission through sexual intercourse is considered.

(b) Susceptible and Infected are removed at a constant natural death rate

(c) Constant emigration rate $m$ is positive for sexually mature susceptible and infective

(d) The model assumes a constant incubation period $\tau>0$ 
(e) The model assumes homogeneous mixing, thus it assumes standard incidence of the form $\lambda_{j}^{n} S_{i}(t-n):=\frac{\beta_{j} c_{i} S_{i}(t-n)\left(I_{j}(t-n)+\eta A_{j}(t-n)\right)}{N_{j}(t-n)}$,

where $n=0, \tau$ and $\beta_{j} c_{i}, i=f, m, i \neq j$ are the average number of adequate contacts of one infective individual per unit time

(f) AIDS cases are not easily recognized in the population because of the use of Highly Active Antiretroviral Treatment (HAARTs) and thus contribute in the spread of the epidemic

The assumptions made above leads to the following differential equations that describe the dynamics of the epidemic

$$
\begin{aligned}
& S_{i}^{\prime}(t)=\rho \Lambda-\beta_{j} c_{i} \frac{S_{i}(t)\left(I_{j}(t)+\eta A_{j}(t)\right)}{N_{j}(t)}-(\mu+m) S_{i}(t), \\
& I_{i}(t)=\int_{t-\tau}^{t} \beta_{j} c_{i} \frac{S_{i}(u)\left(I_{j}(u)+\eta A_{j}(u)\right)}{N_{j}(u)} \exp \{-(\mu+m)(t-u)\} d u, \\
& A_{i}^{\prime}(t)=\beta_{j} c_{i} k \frac{S_{i}(t-\tau)\left(I_{j}(t-\tau)+\eta A_{j}(t-\tau)\right)}{N_{j}(t-\tau)}-(\mu+v) A_{i}(t)
\end{aligned}
$$

where $i=f, m, i \neq j$. In order to analyze the AIDS model in equations (1), the second integral equation of (1) should be written in an equivalent delay differential equation.

Thus the model becomes

$$
\begin{aligned}
& S_{i}^{\prime}(t)=\rho \Lambda-\beta_{j} c_{i} \frac{S_{i}(t)\left(I_{j}(t)+\eta A_{j}(t)\right)}{N_{j}(t)}-(\mu+m) S_{i}(t) \\
& I_{i}^{\prime}(t)=\beta_{j} c_{i} \frac{S_{i}(t)\left(I_{j}(t)+\eta A_{j}(t)\right)}{N_{j}(t)}-\beta_{j} c_{i} k \frac{S_{i}(t-\tau)\left(I_{j}(t-\tau)+\eta A_{j}(t-\tau)\right)}{N_{j}(t-\tau)}-(\mu+m) I_{i}(t) \\
& A_{i}^{\prime}(t)=\beta_{j} c_{i} k \frac{S_{i}(t-\tau)\left(I_{j}(t-\tau)+\eta A_{j}(t-\tau)\right)}{N_{j}(t-\tau)}-(\mu+v) A_{i}(t)
\end{aligned}
$$

where $k=e^{-(\mu+m) \tau}$ is the probability that an infected individual will survive until he or she develops AIDS after time $\tau$ (incubation period) and the parameters $\beta_{i}, c_{i}, \rho, \Lambda, \mu, m$ and $\tau \in \square_{+} ; i=f, m$. System (2) represents the sexually mature age group between 14 and 59 years and it is this age that is responsible for the spread of HIV/AIDS through sexual intercourse. The model (2) has initial conditions at time $t=0$ given by:

$$
\begin{aligned}
& S_{i}(s)=S_{i, 0}(s) \geq 0, \quad I_{i}(s)=I_{i, 0}(s) \geq 0 \quad \forall s \in[-\tau, 0] \\
& \text { with } S_{i}(0)>0, \quad I_{i}(0)>0, \quad A_{i}(0)>0 \quad \text { where } \quad i=f, m
\end{aligned}
$$

Based on biological considerations, the model (2) will be studied in the following region 


$$
\begin{aligned}
& \square_{+0}=\left\{\left(S_{i}, I_{i}, A_{i}, N_{\tau}\right) \mid S_{i} \geq 0, I_{i} \geq 0, A_{i} \geq 0, N_{\tau}(t) \geq 0\right\} \\
& \square_{0}=\left\{\left(S_{i}, I_{i}, A_{i}, N_{\tau}\right) \mid S_{i}>0, I_{i}>0, A_{i}>0, N_{\tau}(t)>0\right\} \\
& \square_{+0}=\left\{\left(S_{i}, I_{i}, A_{i}, N_{\tau}\right) \mid S_{i} \geq 0, I_{i} \geq 0, A_{i} \geq 0, N_{\tau}(t) \geq 0 ; \frac{\Lambda}{(\mu+m)} \geq N_{\tau} \geq \frac{\Lambda}{(\mu+v)}\right\}
\end{aligned}
$$

where $i=f, m$ is positively invariant with respect to the model system (2)

\subsection{Positivity and Boundedness of solutions}

Model (2) describes human population dynamics and therefore it is important to prove that all the state variables $(S(t), I(t)$, and $A(t))$ are non-negative for all time $t$. We show that all solutions of the system (2) with positive initial data will remain positive for all $t>0$ and are bounded in $\mathbf{R}$ Theorem 2.1.

Let the initial data be $S_{i}(s)=S_{i, 0}(s) \geq 0, \quad I_{i}(s)=I_{i, 0}(s) \geq 0, \quad \forall s \in[-\tau, 0) \quad$ with $S_{i, 0}(0)>0, \quad I_{i, 0}(0)>0, \quad A_{i, 0}(0)>0$. Then the solutions $S_{i}(t), I_{i}(t)$ and $A_{i}(t)$ of the system (2) are positive for all $t>0$. For the model system (2), the region $\mathbf{R}$ is positively invariant and all solutions starting in $\mathbf{D}_{\mathbf{- 0}}$ or $\mathbf{D}_{\mathbf{+ 0}}$ approach, enter or stay in $\mathbf{D}_{\mathbf{+} \mathbf{0}}$

Proof

Under the given initial conditions, it is easy to prove that the components of solutions of the system (2), $S_{i}=S_{i}(t) ; i=f, m$ is positive for $t>0$. In this case, the first part of system (2) can be written as

$$
S_{i}^{\prime}(t)=-\beta_{j} c_{i} \frac{S_{i}(t)\left(I_{j}(t)+\eta A_{j}(t)\right)}{N_{j}(t)}-(\mu+m) S_{i}(t) .
$$

If not, we assume a contradiction that $S_{i}(t) ; i=f, m$, is not positive for all $t \geq 0$. By continuity of $S_{i}(t)$ and the fact that $S_{i, 0}(0)>0$, then; $S_{i}(t)<0$ implies that there exist an initial time $T_{0}$ such that $S_{i}\left(T_{0}\right)=0, S_{i}^{\prime}\left(T_{0}\right) \leq 0$ and $S_{i}(t)>0$ for $0<t<T_{0}$

Now integrating (3) from 0 to $T_{0}$, we get

$$
S_{i}(t) \geq S_{i, 0} \exp \left\{\int_{0}^{T_{0}}\left[-\beta_{j} c_{i} \frac{I_{j}(t)+\eta A_{j}(t)}{N_{j}(t)}-(\mu+m)\right] d t\right\}>0 .
$$

This is a contradiction and hence $S_{i}(t)$ is positive for all

$t \geq 0$

Similarly, to show that $I_{i}(t)>0 ; i=f, m$, we assume that there exist a first time $T$, such that $I_{i}(T)=0$ and $I_{i}^{\prime}(T) \leq 0$ and $I_{i}(t)>0$ for all $0<t<T$. In this case, it follows from system (2) that for $T>\tau$ 


$$
I_{i}^{\prime}(t)=\beta_{j} c_{i} \frac{S_{i}(t)\left(I_{j}(t)+\eta A_{j}(t)\right)}{N_{j}(t)}-\beta_{j} c_{i} k \frac{S_{i}(t-\tau)\left(I_{j}(t-\tau)+\eta A_{j}(t-\tau)\right)}{N_{j}(t-\tau)}-(\mu+m) I_{i}(t)>0
$$

which is a contradiction, thus $I_{i}(t)$ remain positive for all $t>0$

Similarly, using the same argument, we can show that $A_{i}(t)$ remain positive for all $t>0$. Thus, we conclude that solutions of the system (2) remain positive for all $t>0$.

Since $N_{T}(t) \geq A_{i}$, then $\Lambda-(\mu+v) N_{T}(t) \leq N_{T}^{\prime}(t) \leq \Lambda-(\mu+m) N_{T}(t)$ implies that $N_{T}(t)$ is bounded and all solutions starting in the region $\mathbf{D}_{+0}$ or $\mathbf{D}_{+0}$ approach, enter or stay in $\mathbf{R}$.

\subsection{Disease-free state and its stability}

We analyze the system (2) by finding its equilibriums and studying their linear stability. The disease-free equilibrium is given by

$$
E_{0}=\left(S_{f}^{0}, S_{m}^{0}, I_{f}^{0}, I_{m}^{0}, A_{f}^{0}, A_{m}^{0}\right)=\left(\frac{\Lambda \rho}{\mu+m}, \frac{\Lambda(1-\rho)}{\mu+m}, 0,0,0,0\right) .
$$

We compute the basic reproduction number, following the next generation operator approach by Dickmann et al. [3] , van Deen Driesche and Watmough [17] approaches in which they define the reproduction number $R_{0}$ as the spectral radius of the next generation operator. Using the given approach, the basic reproduction number $R_{0}$ is defined by

$$
R_{0}=\rho\left(F V^{-1}\right),
$$

where $F$ is non-negative matrix, $V$ is a non-singular $M-$ matrix and $F V^{-1}$ is the next-generation matrix of the model and $\rho(A)$ denotes the spectral radius of matrix $A$. For the model system (2) we obtain

$$
F=\left(\begin{array}{lc}
0 & \frac{\beta_{m} c_{f}(1-k) S_{f}^{0}}{S_{m}^{0}} \\
\frac{\beta_{f} c_{m}(1-k) S_{m}^{0}}{S_{f}^{0}} & 0
\end{array}\right) ; V=\left(\begin{array}{rr}
\mu+m & 0 \\
0 & \mu+m
\end{array}\right) \text { and } V^{-1}=\left(\begin{array}{ll}
\frac{1}{\mu+m} & 0 \\
0 & \frac{1}{\mu+m}
\end{array}\right)
$$

And the basic reproduction number is

$$
R_{0}=\frac{\sqrt{\beta_{f} \beta_{m} c_{f} c_{m}}(1-k)}{(\mu+m)}=\sqrt{R_{0 f} R_{0 m}}
$$

where $R_{0 f}=\frac{\beta_{f} c_{f}(1-k)}{(\mu+m)}$ and $R_{0 m}=\frac{\beta_{m} c_{m}(1-k)}{(\mu+m)}$ are females and males contribution to the basic reproduction number, $R_{0}$ respectively. In this respect, we can define $R_{0}$ as the geometric mean of $R_{0 f}$ and $R_{0 m}$ 


\subsection{Global stability of disease-free equilibrium}

\section{Theorem 2.2.}

If $R_{0}<1$, the disease-free equilibrium is globally asymptotically stable and if $R_{0}>1$, this equilibrium is unstable.

\section{Proof}

We first rewrite $I_{i}(t)$ and $I_{m}(t)$ from system (1) as

$$
I_{i}(t)=\int_{t-\tau}^{t} \beta_{m} c_{f} \frac{\left(N_{f}(u)-I_{i}(u)\right) I_{m}(u)}{N_{m}(u)} \exp \{-(\mu+m)(t-u)\} d u
$$

and

$$
I_{m}(t)=\int_{t-\tau}^{t} \beta_{f} c_{m} \frac{\left(N_{m}(u)-I_{m}(u)\right) I_{i}(u)}{N_{f}(u)} \exp \{-(\mu+m)(t-u)\} d u
$$

Where $\quad N_{f}(u)=S_{f}(u)+I_{f}(u)+A_{f}(u) \quad$ and $\quad N_{m}(u)=S_{m}(u)+I_{m}(u)+A_{m}(u) \quad$ Using substitution $x=t-u$, taking the lim sup of both sides of equation (8) and applying the fact that $\limsup \int f n \leq \int \limsup f n$, we get

$$
\begin{aligned}
\limsup _{t \rightarrow \infty} I_{i}(t) & =\limsup _{t \rightarrow \infty} \int_{t-\tau}^{t} \beta_{m} c_{f} \frac{\left(N_{f}(t-x)-I_{i}(t-x)\right) I_{m}(t-x)}{N_{m}(t-x)} \exp \{-(\mu+m) x\} d x \\
& \leq \int_{t-\tau}^{t} \beta_{m} c_{f} \limsup _{t \rightarrow \infty} \frac{\left(N_{f}(t-x)-I_{i}(t-x)\right)}{N_{m}(u)} \limsup _{t \rightarrow \infty} I_{m}(t-x) \exp \{-(\mu+m) x\} d x \\
& \leq \beta_{m} c_{f} \limsup _{t \rightarrow \infty} \frac{N_{f}(t)-I_{i}(t)}{N_{m}(u)} \limsup _{t \rightarrow \infty} I_{m}(t) \int_{0}^{\tau} e^{-(\mu+m) x} d x \\
& \leq \frac{\beta_{m} c_{f}\left(1-e^{-(\mu+m) \tau}\right)}{\mu+m} \limsup _{t \rightarrow \infty} I_{m}(t) \\
& \left(\frac{\beta_{m} c_{f}\left(1-e^{-(\mu+m) \tau}\right)}{\mu+m} \limsup _{t \rightarrow \infty}\left(\int_{t-\tau}^{t} \beta_{f} c_{m} \frac{\left(N_{m}(t-x)-I_{m}(t-x)\right) I_{i}(t-x)}{N_{f}(t-x)} \exp \{-(\mu+m) x\} d x\right)\right)
\end{aligned}
$$

(From (9) and making substitution $x=t-u$ ) 


$$
\begin{aligned}
& \leq \frac{\beta_{m} c_{f}\left(1-e^{-(\mu+m) \tau}\right)}{\mu+m} \int_{t-\tau}^{t} \limsup _{t \rightarrow \infty} \beta_{f} c_{m} \frac{\left(N_{m}(t-x)-I_{m}(t-x)\right)}{N_{f}(t-x)} \limsup _{t \rightarrow \infty} I_{i}(t-x) \exp \{-(\mu+m) x\} d x \\
& \leq \frac{\beta_{m} \beta_{f} c_{f} c_{m}\left(1-e^{-(\mu+m) \tau}\right)}{\mu+m} \limsup _{t \rightarrow \infty} \frac{\left(N_{m}(t)-I_{m}(t)\right)}{N_{f}(t)} \limsup _{t \rightarrow \infty} I_{i}(t) \int_{t-\tau}^{t} \exp \{-(\mu+m) x\} d x \\
& \leq \frac{\beta_{m} \beta_{f} c_{f} c_{m}\left(1-e^{-(\mu+m) \tau}\right)\left(1-e^{-(\mu+m) \tau}\right)}{(\mu+m)(\mu+m)} \limsup _{t \rightarrow \infty} I_{i}(t)=R_{0}^{2} \limsup _{t \rightarrow \infty} I_{i}(t)
\end{aligned}
$$

Thus if $\frac{\beta_{m} \beta_{f} c_{f} c_{m}\left(1-e^{-(\mu+m) \tau}\right)\left(1-e^{-(\mu+m) \tau}\right)}{(\mu+m)(\mu+m)}=R_{0}^{2}<1$, we have a strict inequality

$\limsup _{t \rightarrow \infty} I_{m}(t)<\limsup _{t \rightarrow \infty} I_{m}(t) ;$ unless $\limsup _{t \rightarrow \infty} I_{m}(t)=0$.

From the former case, we have $\limsup I_{i}(t)<R_{0}^{2} \limsup I_{i}(t)$ and from the latter case, we have $\limsup I_{m}(t)<R_{0}^{2} \limsup I_{m}(t)$ for $R_{0}<1$ which are strict inequalities and contradictions, we conclude that the disease-free equilibrium is globally asymptotically stable if $R_{0}<1$

\subsection{Endemic equilibrium and its stability}

The endemic equilibrium for the system (2) $E_{e}=\left(S_{f}^{e}, S_{m}^{e}, I_{f}^{e}, I_{m}^{e}, A_{f}^{e}, A_{m}^{e}\right)$ is $\left(\frac{\left(\mu+m+\beta_{f} c_{m}(1-k)\right) I_{f}^{e}}{\left(R_{0}^{2}-1\right)(\mu+m)}, \frac{\left(\mu+m+\beta_{m} c_{f}(1-k)\right) I_{m}^{e}}{\left(R_{0}^{2}-1\right)(\mu+m)}, I_{f}^{e}, I_{m}^{e}, \frac{(\mu+m) k I_{f}^{e}}{(1-k)(\mu+v)}, \frac{(\mu+m) k I_{m}^{e}}{(1-k)(\mu+v)}\right)$

where,

$$
\begin{aligned}
& I_{f}^{e}=\frac{\Lambda \rho(1-k)(\mu+m)(\mu+m)\left(\left(R_{m}^{2}-1\right)\right)}{\left(\left((\mu+m) \beta_{m} c_{f}(k-1)\left(\mu+m+\beta_{m} c_{f}\right)+k\left(m^{2}+\mu+\beta_{f} c_{m}(1-k) \mu+\beta_{m} c_{f}\right)\right)+m\left(2 \mu+\beta_{f} \beta_{m} c_{f} c_{m}(1-k)\right)\right)} \\
& I_{m}^{e}=\frac{\Lambda(1-\rho)(k-1)(\mu+m)(\mu+m)\left(\left(R_{m}^{2}-1\right)\right)}{\left(\left((\mu+m) \beta_{m} c_{f}(k-1)\left(\mu+m+\beta_{f} c_{m}\right)+k\left(m^{2}+\mu+\beta_{m} c_{f}(1-k) \mu+\beta_{f} c_{m}\right)\right)+m\left(2 \mu+\beta_{f} \beta_{m} c_{f} c_{m}(1-k)\right)\right)}
\end{aligned}
$$

since the model (2) monitors human population, the endemic equilibrium point $\left(E_{e}\right)$ should always be positive. We can conclude from expressions for $I_{f}^{e}$ and $I_{m}^{e}$ that the endemic equilibrium is positive when $R_{0}>1$. This result can be summarized in the following theorem:

\section{Theorem 2. 3.}

The endemic equilibrium $E_{e}$ exists and is positive if $R_{0}>1$ 


\section{Peer- Educators' Campaign}

We analyze the effects of Peer-educators campaign as a single strategy for HIV/AIDS prevention. We define peer-education campaign as the counselling of individuals to engage in safe sex or low risk sexual behaviours.

High risk behavior includes sex after getting "high", having many sexual patterns, having unprotected sexual relations, polygamy, having sex with commercial sex-workers just to mention but a few. Low risk behavior includes any non-risk behavior e. g delayed sexual; debut, being faithful with one partner, always using a condom among others. It is believed that the most effective counselling session is between a trained person and a client of almost the same age. This call for the need to have peer-counsellors instead of public health campaign

In this context, the following additional parameters are used and assumptions are made to model the effects of peer-education campaign

\subsection{Assumptions and parameters}

(i) the model in equation (1) is modified to have high and low risk susceptible, high and low risk infected and high and low risk AIDS cases denoted $S_{i 1}$ and $S_{i 2} ; I_{i 1}$ and $I_{i 2}$ and $A_{i 1}$ and $A_{i 2}$ respectively, where $i=f, m$ and the subscript 1,2 denote high-risk and low-risk sexual activities respectively.

(ii) Individuals in category $S_{i 1}$ are educated and transfer to low-risk susceptible class $S_{i 2}$ at a rate $\omega_{i}$.

(iii) Upon becoming infected with HIV, a proportion $\pi_{i}$ of educated individuals enter $I_{i 2}$ and the complementary proportion $\left(1-\pi_{i}\right)$ enter the high-risk class $I_{i 1}$

(iv) Individuals in $A_{i 1}$ move to the low risk AIDS classes $A_{i 2}$ at a constant rate of $a_{i}$ due to peereducational campaign.

(v) High risk infected individuals progress to AIDS stage at a rate $\lambda_{j}^{\tau} \pi_{i} k S_{i}(t-\tau)$ and low risk infected individuals progress to AIDS stage at a rate $\lambda_{j}^{\tau}\left(1-\pi_{i}\right) k S_{i}(t-\tau)$.

With the above additional parameters and assumptions, the model in equation (1) becomes 


$$
\begin{aligned}
& S_{i 1}^{\prime}(t)=\rho \Lambda-\beta_{j} c_{i} \frac{S_{i 1}(t)\left(I_{j 1}(t)+\eta A_{j 1}(t)\right)}{N_{j 1}(t)}-\left(\omega_{f}+\mu+m\right) S_{i 1}(t), \\
& S_{i 2}^{\prime}(t)=\rho \Lambda-\beta_{j} c_{i} \frac{S_{i 2}(t)\left(I_{j 2}(t)+\eta A_{j 2}(t)\right)}{N_{j 2}(t)}-\left(\omega_{f}+\mu+m\right) S_{i 2}(t) \\
& I_{i 1}(t)=\int_{t-\tau}^{\tau} \beta_{j} c_{i} \frac{S_{i 1}(u)\left(I_{j 1}(u)+\eta A_{j 1}(u)\right)}{N_{j 1}(u)} \exp \{-(\mu+m)(t-u)\} d u, \\
& I_{i 2}(t)=\int_{t-\tau}^{\tau} \beta_{j} c_{i} \frac{S_{i 2}(u)\left(I_{j 2}(u)+\eta A_{j 2}(u)\right)}{N_{j 2}(u)} \exp \{-(\mu+m)(t-u)\} d u \\
& A_{i 1}^{\prime}(t)=a_{f} \pi_{f} \beta_{j} c_{i} k \frac{S_{i 1}(t-\tau)\left(I_{j 1}(t-\tau)+\eta A_{j 1}(t-\tau)\right)}{N_{j 1}(t-\tau)}-\left(a_{f}+\mu+v\right) A_{i 1}(t) \\
& A_{i 2}^{\prime}(t)=\beta_{j} c_{i} k \frac{S_{i 2}(t-\tau)\left(I_{j 2}(t-\tau)+\eta A_{j 2}(t-\tau)\right)}{N_{j 2}(t-\tau)}+a_{f} A_{f 1}(t)-(\mu+v) A_{i 2}(t)
\end{aligned}
$$

where $i=f, m, \quad i \neq j$. In order to analyze the AIDS model in equation (1), the third and fourth parts of (13) should be written in an equivalent delay differential equation, thus the model becomes

$$
\begin{aligned}
& S_{i 1}^{\prime}(t)=\rho \Lambda-\beta_{j} c_{i} \frac{S_{i 1}(t)\left(I_{j 1}(t)+\eta A_{j 1}(t)\right)}{N_{j 1}(t)}-\left(\omega_{f}+\mu+m\right) S_{i 1}(t), \\
& S_{i 2}^{\prime}(t)=\rho \Lambda-\beta_{j} c_{i} \frac{S_{i 2}(t)\left(I_{j 2}(t)+\eta A_{j 2}(t)\right)}{N_{j 2}(t)}-\left(\omega_{f}+\mu+m\right) S_{i 2}(t) \\
& I_{i 1}^{\prime}(t)=\beta_{j} c_{i} \frac{S_{i 1}(t)\left(I_{j 1}(t)+\eta A_{j 1}(t)\right)}{N_{j 1}(t)}-\beta_{j} c_{i} k \frac{S_{i 1}(t-\tau)\left(I_{j 1}(t-\tau)+\eta A_{j 1}(t-\tau)\right)}{N_{j 1}(t-\tau)}-(\mu+m) I_{i 1}(t) \\
& I_{i 2}^{\prime}(t)=\beta_{j} c_{i} \frac{S_{i 2}(t)\left(I_{j 2}(t)+\eta A_{j 2}(t)\right)}{N_{j 2}(t)}-\beta_{j} c_{i} k \frac{S_{i 2}(t-\tau)\left(I_{j 2}(t-\tau)+\eta A_{j 2}(t-\tau)\right)}{N_{j 2}(t-\tau)}-(\mu+m) I_{i 2}(t) \\
& A_{i 1}^{\prime}(t)=a_{f} \pi_{f} \beta_{j} c_{i} k \frac{S_{i 1}(t-\tau)\left(I_{j 1}(t-\tau)+\eta A_{j 1}(t-\tau)\right)}{N_{j 1}(t-\tau)}-\left(a_{f}+\mu+v\right) A_{i 1}(t) \\
& A_{i 2}^{\prime}(t)=\beta_{j} c_{i} k \frac{S_{i 2}(t-\tau)\left(I_{j 2}(t-\tau)+\eta A_{j 2}(t-\tau)\right)}{N_{j 2}(t-\tau)}+a_{f} A_{f 1}(t)-(\mu+v) A_{i 2}(t)
\end{aligned}
$$

where $k=\exp \{-(\mu+m) \tau\}$ is the probability that an infected individual will survive until he or she develops AIDS after time $\tau$ (incubation period). 


\subsection{Equilibrium points and stability}

The system (14) has the disease-free equilibrium

$E_{0}=\left(S_{f 1}^{0}, S_{m 1}^{0}, I_{f 1}^{0}, I_{m 1}^{0}, A_{f 1}^{0}, A_{m 1}^{0}\right)=\left(\frac{\Lambda \rho}{\mu+m}, \frac{\Lambda(1-\rho)}{\mu+m}, 0,0,0,0\right)$ and the endemic equilibrium point (EEP) of this model will be

$$
\begin{aligned}
& E_{e}=\left(S_{f 1}^{e}, S_{m 1}^{e}, I_{f 1}^{e}, I_{m 1}^{e}, A_{f 1}^{e}, A_{m 1}^{e}\right) \\
& \quad=\left(\frac{\Lambda \rho}{\mu+v+\omega_{f}+\lambda_{m}^{e}}, \frac{\Lambda(1-\rho)}{\mu+v+\omega_{f}+\lambda_{f}^{e}}, \frac{(1-k) \pi_{f} \Lambda \rho \lambda_{m}^{e}}{\left((\mu+m) \mu+v+\omega_{f}+\lambda_{m}^{e}\right)}, \frac{(1-k) \pi_{f} \Lambda(1-\rho) \lambda_{f}^{e}}{\left((\mu+m) \mu+v+\omega_{f}+\lambda_{f}^{e}\right)},\right. \\
& \left.\frac{k\left(b_{f}\left(1-\pi_{f}\right)+a_{f} \pi_{f}\right) \rho \Lambda \lambda_{m}^{e}}{\left(\mu+v+\alpha_{f}\right)\left(\mu+v+\omega_{f}+\lambda_{m}^{e}\right)}, \frac{k\left(b_{m}\left(1-\pi_{m}\right)+a_{m} \pi_{m}\right)(1-\rho) \Lambda \lambda_{f}^{e}}{\left(\mu+v+\alpha_{f}\right)\left(\mu+v+\omega_{f}+\lambda_{f}^{e}\right)}\right) \\
& \text { where } \lambda_{j}^{e}=\frac{\beta_{j} c_{i}\left(I_{j 1}^{e}+A_{j 1}^{e}\right)}{N_{j 1}^{e}}
\end{aligned}
$$

\subsection{The basic reproduction number}

In obtaining the basic reproduction number $R_{f 1}$ for the system (14), we consider a single newly infected high risk male entering the disease-free population at equilibrium. The individual is still present and infectious at $t<\tau$ with probability $\exp \{-(\mu+m) t\}$ and in this case infects females at rate $\pi_{f} \beta_{m} c_{f} \frac{S_{f 1}^{0}}{S_{m 1}^{0}}$.

Hence, the expected number of females infected by this high risk male is approximately

$$
R_{f 1}=\int_{0}^{\tau} \pi_{f} \beta_{m} c_{f} \frac{S_{f 1}^{0}}{S_{m 1}^{0}} e^{-(\mu+m) t} d t=\frac{\pi_{f} \beta_{m} c_{f} S_{f 1}^{0}}{(\mu+m) S_{m 1}^{0}}(1-k)
$$

Similarly, the expected number of males infected by each of these females is approximately

$$
R_{m 1}=\int_{0}^{\tau} \pi_{m} \beta_{f} c_{m} \frac{S_{m 1}^{0}}{S_{f 1}^{0}} e^{-(\mu+m) t} d t=\frac{\pi_{m} \beta_{f} c_{m} S_{m 1}^{0}}{(\mu+m) S_{f 1}^{0}}(1-k) .
$$

Considering the high risk female AIDS case entering the disease-free population at equilibrium in a similar fashion we have,

$$
R_{f 2}=\int_{0}^{\infty}\left(a_{f} \pi_{m}+\beta_{f}\left(1-\pi_{f}\right)\right) \beta_{m} c_{f} k \frac{S_{f 1}^{0}}{S_{m 1}^{0}} e^{-\left(a_{f}+\mu+v\right) t} d t=\frac{\left(a_{f} \pi_{m}+\beta_{f}\left(1-\pi_{f}\right)\right) \beta_{m} c_{f} k S_{f 1}^{0}}{\left(\alpha_{f}+\mu+v\right) S_{m 1}^{0}} .
$$

In the same way we find

$$
R_{m 2}=\frac{\left(a_{m} \pi_{f}+\beta_{m}\left(1-\pi_{m}\right)\right) \beta_{f} c_{m} k S_{m 1}^{0}}{\left(\alpha_{m}+\mu+v\right) S_{f 1}^{0}}
$$

The average expected number of secondary cases per generation produced by each infectious male is $\sqrt{R_{f 1} R_{m 1}+R_{f 2} R_{m 1}+R_{f 1} R_{m 2}+R_{f 2} R_{m 2}}$. 
Interpreting generation as alternating male and female cases and the expected average number of secondary cases per generation produced by each infectious female

$$
\sqrt{R_{f 1} R_{m 1}+R_{f 2} R_{m 1}+R_{f 1} R_{m 2}+R_{f 2} R_{m 2}},
$$

the reproduction number $R_{0}$ for the model (14) can be written as

$$
R_{0}=\left(\beta_{f} \beta_{m} c_{f} c_{m}\left(\begin{array}{c}
\frac{\pi_{f} \pi_{m}(1-k)^{2}}{(m+\mu)^{2}}+\frac{k(1-k)}{(v+\mu)}\left[\frac{\pi_{m}\left(b_{f}\left(1-\pi_{f}\right)+a_{f} \pi_{f}\right)}{\left(\mu+v+\alpha_{f}\right)}+\frac{\pi_{f}\left(b_{m}\left(1-\pi_{m}\right)+a_{m} \pi_{m}\right)}{\left(\mu+v+\alpha_{m}\right)}\right]+ \\
\frac{k^{2}\left(b_{f}\left(1-\pi_{f}\right)+a_{f} \pi_{f}\right)\left(b_{m}\left(1-\pi_{m}\right)+a_{m} \pi_{m}\right)}{\left(\mu+v+\alpha_{f}\right)\left(\mu+v+\alpha_{m}\right)}
\end{array}\right)\right)^{\frac{1}{2}}
$$

\subsection{Effects of Peer- Educational campaign on $R_{0}$}

We use the computed $R_{0}$ to estimate the effects of peer-educational campaign in controlling HIV/AIDS in a community for the following cases,

(i) Case 1: When there is completely no educational campaigns, $\pi_{i}=a_{i}=b_{i}=1$ and $\alpha_{i}=0$. for this case we have

$$
\lim _{\pi_{i}, a_{i}, b_{i} \rightarrow 1} R_{0}=R_{1}=\sqrt{\beta_{f} \beta_{m} c_{f} c_{m}\left(\frac{(1-k)^{2}}{(m+\mu)^{2}}+\frac{k(1-k)}{(v+\mu)}\left[\frac{1}{\mu+v}+\frac{1}{\mu+v}\right]+\frac{k^{2}}{(\mu+v)(\mu+v)}\right)},
$$

where $R_{1}$ is the reproductive number when there is no educational campaign.

We have that $R_{1}>R_{0}$ suggesting that lack of educational campaigns in communities with HIV/AIDS, results in an increase in the number of secondary infections.

(ii) Case 2: When there is effective educational campaigns, $\pi_{i}=a_{i}=b_{i}=0$ and $\alpha_{i}>0$. For this case we shall have $\lim _{\pi_{i}, a_{i}, b_{i} \rightarrow 0} R_{0}=R_{1}=0$.

We have the effective peer-group educational campaigns in a community, the number of secondary infections is reduced to zero thus the effective educational campaigns help slow or eradicate the epidemic if properly implemented in communities affected by the epidemic

\section{Situation in Rwanda}

\subsection{Evolution of HIV/AIDS in Rwanda}

The first cases of HIV in Rwanda have been reported in 1980. In this year, 4257 cases have been recorded. Since then, the evolution of HIV increased like in other developing countries. The following figure illustrates the evolution of HIV in Rwanda. The highest rate of HIV was reported in 1999. 


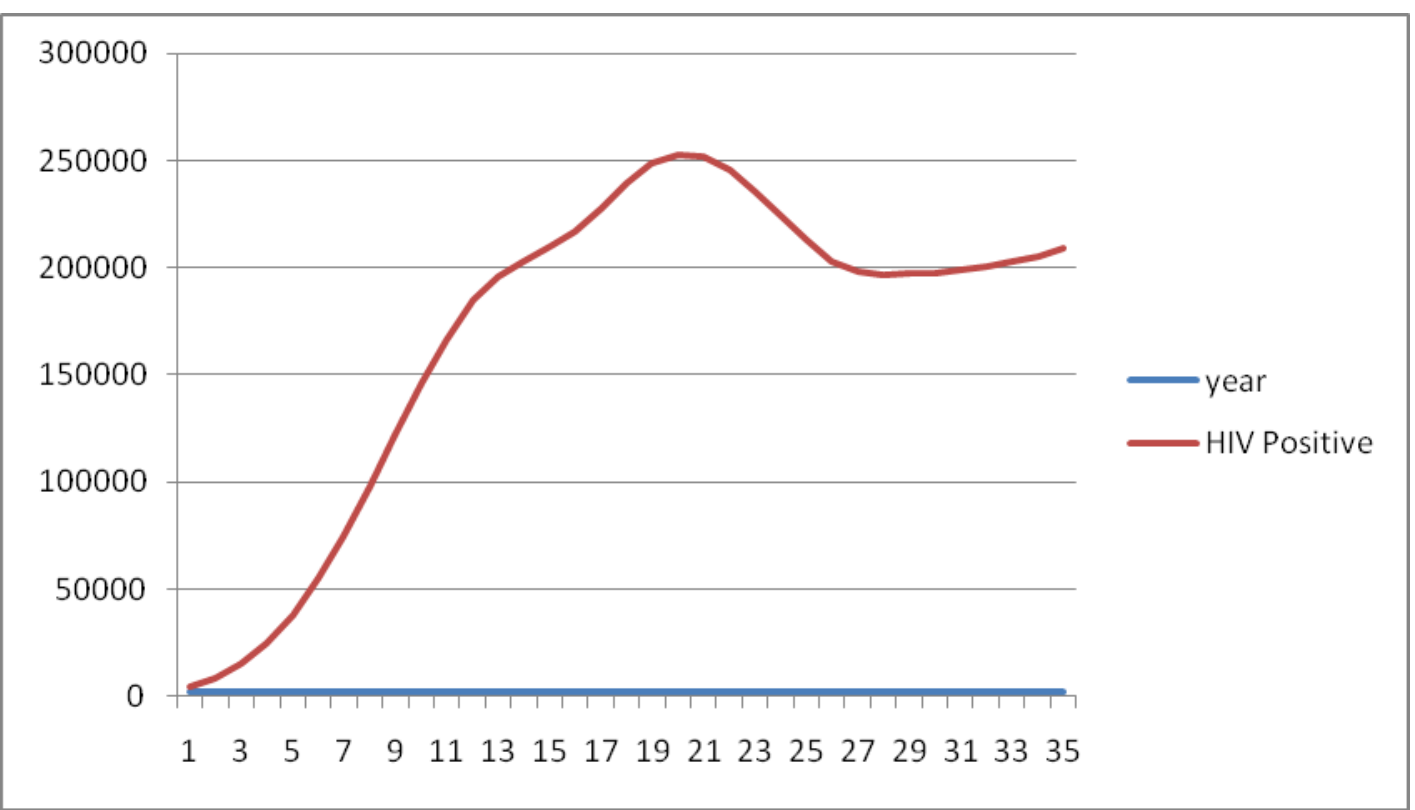

Figure 1: HIV evolution in Rwanda 1980-2014

Considering the number of infectious for each of the mentioned year, and considering the transition matrix for both male and female, the basic reproduction number is estimated to 3.92 for male and 3.25 for female.

In 2010, a random sample of 13,522 individuals were tested for HIV in the whole country; the following table summarizes the information about that survey

Table 2: Factors for spreading HIV in Rwanda

\begin{tabular}{|c|c|c|c|}
\hline \multirow{8}{*}{$\begin{array}{l}\text { Back Ground Cnaracteristics } \\
\text { PROVINCE } \\
\text { North } \\
\text { South } \\
\text { East } \\
\text { West } \\
\text { Kigali City } \\
\text { Total }\end{array}$} & Number (\%) & \multicolumn{2}{|c|}{ HIV Status } \\
\hline & \multirow{7}{*}{$\begin{array}{l}2069 \\
3186 \\
3273 \\
2983 \\
2011 \\
\mathbf{1 3 5 2 2}\end{array}$} & Positive & Negative \\
\hline & & $66(3.19 \%)$ & $2003(96.81 \%)$ \\
\hline & & $99(3.11 \%)$ & $3087(96.89 \%)$ \\
\hline & & $93(2.84 \%)$ & $3180(97.16 \%)$ \\
\hline & & $110(3.69 \%)$ & $2873(96.31 \%)$ \\
\hline & & $61(3.03 \%)$ & $1950(96.97 \%)$ \\
\hline & & $429(3.17 \%)$ & 13093(96.83\%) \\
\hline PLACE OF RESIDENCE & & & \\
\hline Urban & 2482 & $357(14.38 \%)$ & $2125(85.62 \%)$ \\
\hline Rural & 11040 & $72(0.01 \%)$ & $10968(99.99 \%)$ \\
\hline Total & 13522 & $429(3.17 \%)$ & $13093(96.83 \%)$ \\
\hline
\end{tabular}




\begin{tabular}{|l|l|l|l|}
\hline AGE GROUP & & & \\
$15-19$ years & 3065 & $90(2.93 \%$ & $2975(97.06 \%$ \\
$20-24$ years & 2463 & $91(3.70 \%$ & $237296.3 \%$ \\
$25-29$ years & 2237 & $59(2.64 \%)$ & $2178(97.36 \%)$ \\
$30-34$ years & 1562 & $48(3.08 \%)$ & $1514(96.92 \%)$ \\
$35-39$ years & 1041 & $30(2.88 \%)$ & $1011(97.12 \%)$ \\
$40-44$ years & 933 & $29(3.11 \%)$ & $904(96.89 \%)$ \\
$45-49$ years & 869 & $35(4.03 \%)$ & $834(95.97 \%)$ \\
$50-54$ years & 806 & $31(3.85 \%)$ & $775(96.15 \%)$ \\
$55-59$ years & 545 & $16(2.94 \%)$ & $529(97.06 \%)$ \\
Total & $\mathbf{1 3 5 2 2}$ & $\mathbf{4 2 9}(\mathbf{3 . 1 7})$ & $\mathbf{1 3 0 9 3 ( 9 6 . 8 3 \% )}$ \\
\hline EDUCATION & & & \\
No education & 1734 & $47(2.71 \%$ & $1687(97.29 \%)$ \\
Primary & 8777 & $303(3.45 \%$ & $8474(64.7 \%)$ \\
Secondary & 2648 & $61(14.2 \%)$ & $2587(19.8 \%)$ \\
Higher & 363 & $18(4.2 \%)$ & $345(2.6 \%)$ \\
Total & $\mathbf{1 3 5 2 2}$ & $\mathbf{4 2 9}$ & $\mathbf{1 3 0 9 3}$ \\
\hline GENDER & & $359(3.22 \%)$ & $10799(96.78 \%)$ \\
Male & 11158 & $\mathbf{7 0}(2.96 \%)$ & $2294(87.04 \%)$ \\
Female & 2364 & $\mathbf{4 2 9}(\mathbf{3 . 1 7 \%})$ & $\mathbf{1 3 0 9 3 ( 9 6 . 8 3 \% )}$ \\
\hline Total & $\mathbf{1 3 5 2 2}$ &
\end{tabular}

Among these factors, only the education factor was significant. That is why a great attention has been taken to this factor in order to reduce the speed of spreading HIV in Rwanda. First of all "clubs against HIV' have been organized both in schools and out of the schools for youth sensitization. 23 Youth-friend centers are operational. The main objective of these clubs is to sensitize the youth to the danger of this endemic. To assess the results obtained from this policy, A voluntary counseling and testing has been conducted in 2012 and 3,041,056 people were tested for HIV status and $98 \%$ knew their status. The percentage of HIV positive was found to be around $3 \%$. The stability of this rate is due to the extra effort made to sensitize the population about this problem. Now, a new policy is in experimentation where once a week people in the village are meeting in the so called "evening of parents". One of the topics to discuss in these meetings is the transmission of HIV this will help people especially in rural area and for non educated people to know about this endemic. The achievement of this policy will constitute the basis of peereducation campaign against the transmission of HIV. The big challenge of this policy is that the men don't want to attend these meetings.

\subsection{Numerical Simulation}

The parameters that we use for simulations of the models have been obtained from Treatment and Research AIDS Center (TRAC) net and Rwanda Biomedical center (RBC), some of them are assumed and others estimated. 
Table 2. Parameters for the sex- structured HIV/AIDS model.

\begin{tabular}{|l|l|l|}
\hline Parameter & Symbol & Value \\
\hline Birth rate into sexually mature age group & $\Lambda$ & 0.03 \\
\hline Proportion of mature female & $\rho$ & 0.52 \\
\hline Adult natural death & $\mu$ & 0.4 \\
\hline AIDS- related death & $v$ & 0.33 \\
\hline Emigration rate & $m$ & 0.2 \\
\hline Average incubation period & $\tau$ & 8 \\
\hline $\begin{array}{l}\text { Probability of transmission of HIV when there is no } \\
\text { protection }\end{array}$ & $\left(\beta_{f}, \beta_{m}\right)$ & $(0.49,0.52)$ \\
\hline Number of sexual exposures & $\left(c_{f}, c_{m}\right)$ & $(20,29)$ \\
\hline The basic reproduction number & $\left(R_{0}, R_{f}, R_{m}\right)$ & $(3.92,3.65,4.21)$ \\
\hline $\begin{array}{l}\text { the sexual contact rate of a susceptible with an AIDS } \\
\text { case }\end{array}$ & $\eta$ & 0,03 \\
\hline $\begin{array}{l}\text { Proportion of educated people } \\
\text { Rate of individual in } \mathrm{S}_{\mathrm{i} 1 \text { who move to the low risk }}\end{array}$ & $\left(\omega_{f}, \pi_{m}\right)$ & $(0.5,0.5)$ \\
\hline $\begin{array}{l}\text { Rass } \mathrm{S}_{\mathrm{i} 2} \text { due to the peer- education campaign } \\
\text { class } \mathrm{A}_{\mathrm{i} 2} \text { due to the peer- education campaign }\end{array}$ & $(0.4,0.3)$ \\
\hline
\end{tabular}

Taking $\mathrm{S}=0.35, \mathrm{I}=0.10$ and $\mathrm{A}=0.05$ as initial data the figure2 represents the sex structured dynamics of HIV/AIDS in a population without any intervention, using parameters defined in the table 2 


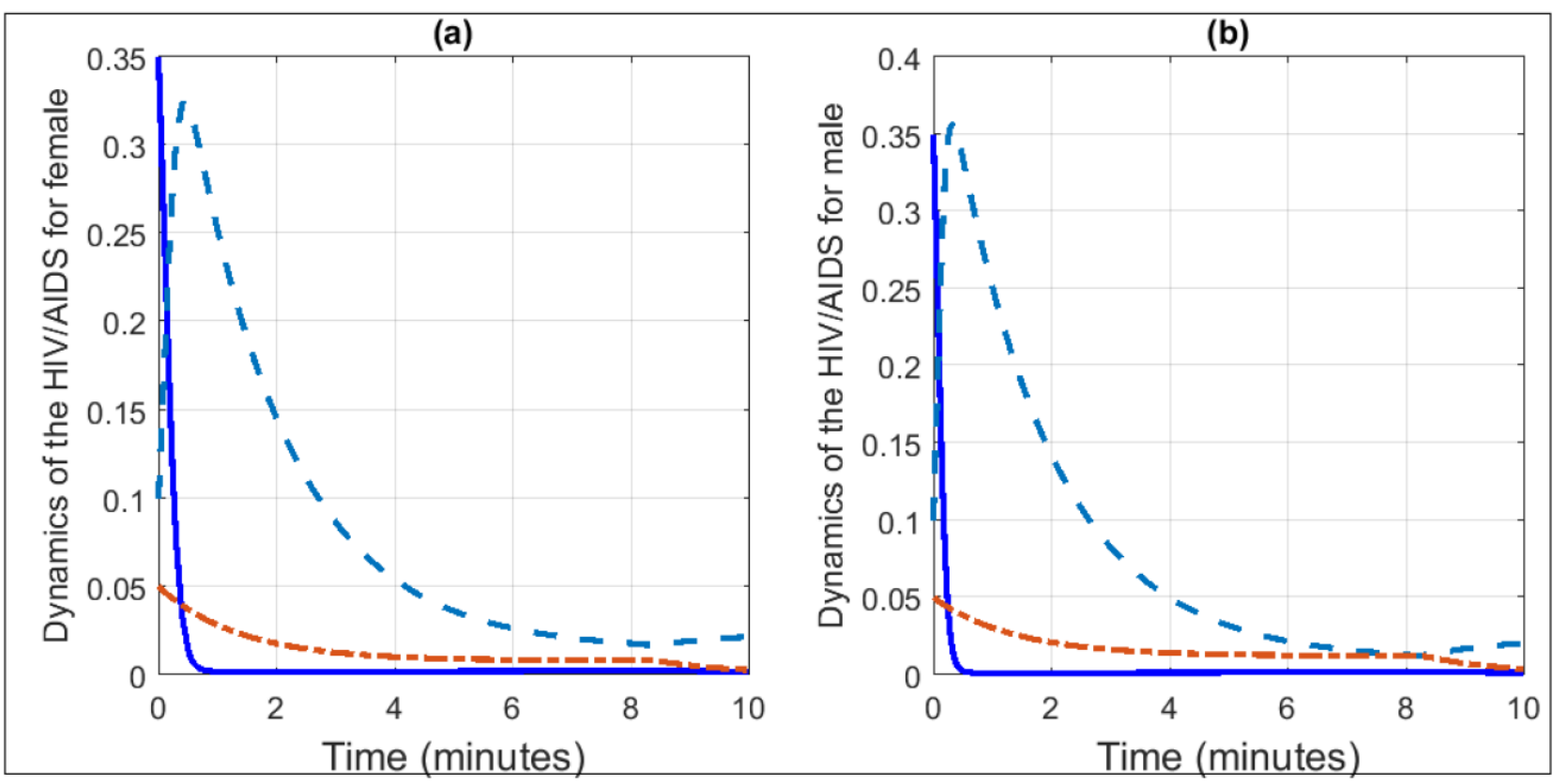

Figure2. General dynamics of HIV/AIDS without any intervention for female (a) and male (b). The solid line shows susceptible (S), the dashed line illustrates infected (I) and the dashdot line represents AIDS cases (A) respectively..

The figure 3 and figure 4 represent the simulation of the dynamics of HIV/AIDS with the effect of peer-education campaign, assuming the following initial data $S_{f 1}=S_{m 1}=0.50, S_{f 2}=S_{m 2}=0.35, I_{f 1}=I_{m 1}=0.20$

$I_{f 2}=I_{m 2}=0.15, A_{f 1}=A_{m 1}=0.10, A_{f 2}=A_{m 2}=0.05$

and the parameters defined in the table 2 .

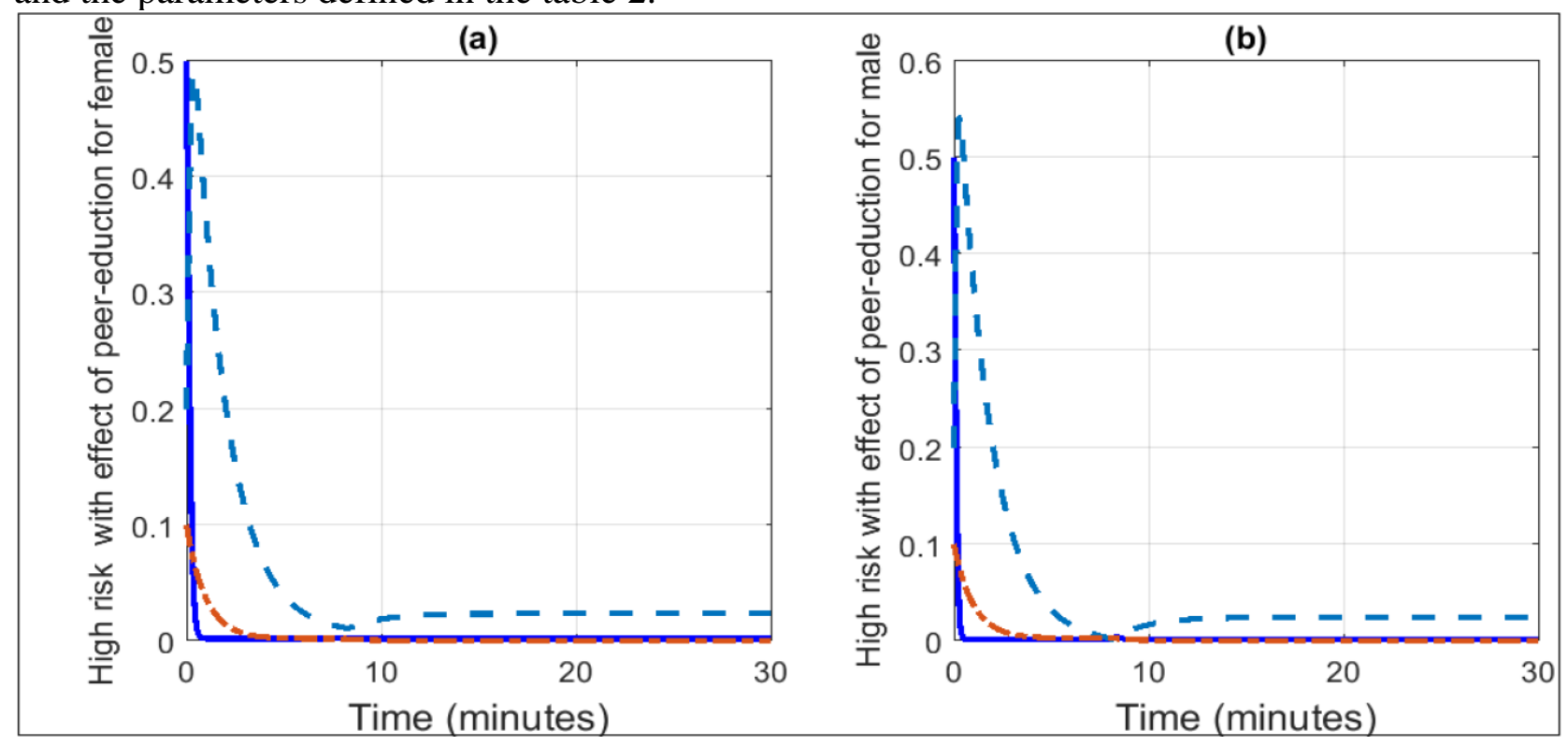


Figure 3. General dynamics of low risk of HIV/AIDS with the peer-education campaign for female (a) and male (b). The solid line shows susceptible (S), the dashed line illustrates infected (I) and the dashdot line represents AIDS cases (A) respectively.

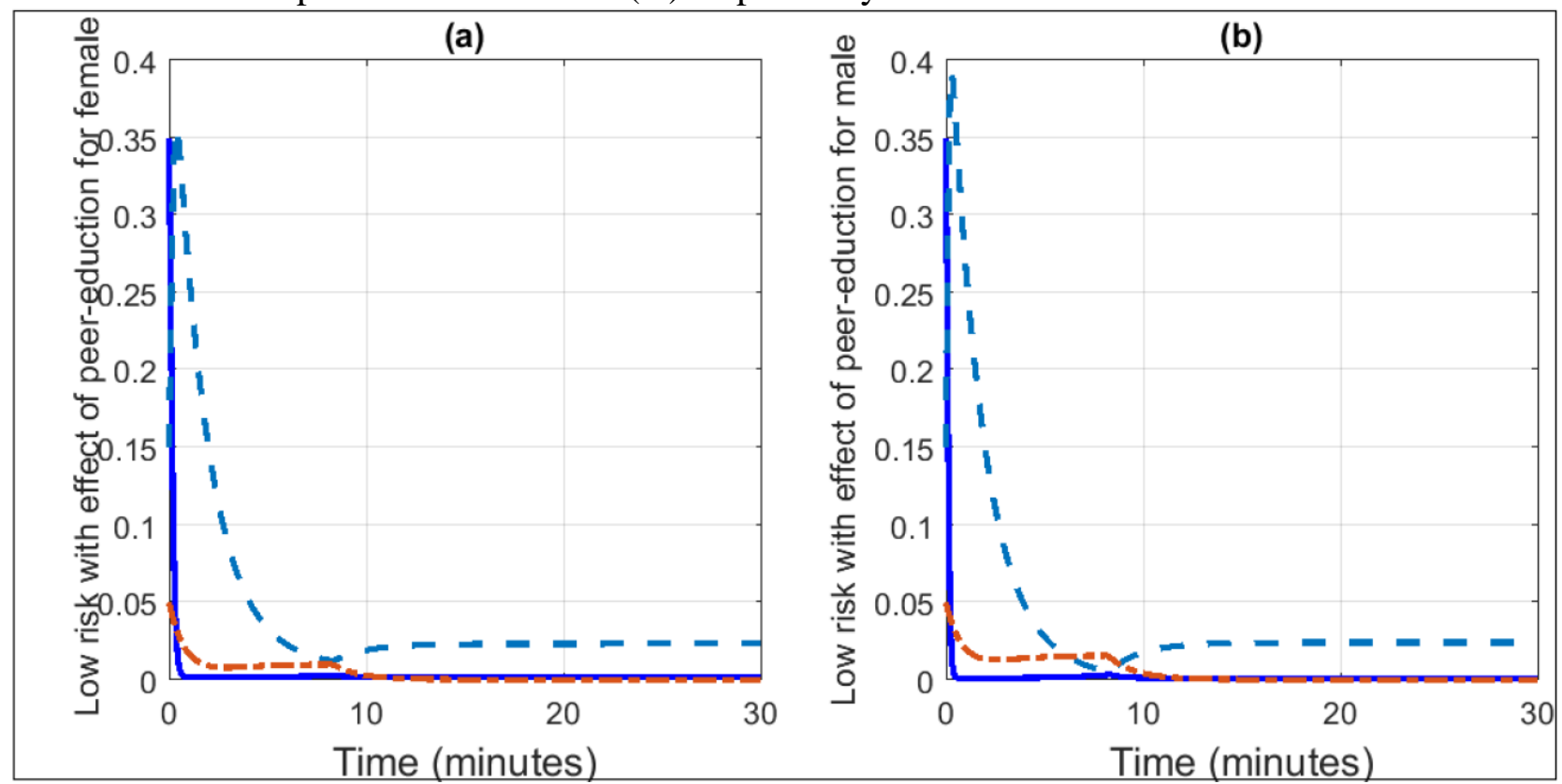

Figure 4. General dynamics of low risk of HIV/AIDS with the peer-education campaign for female (a) and male (b). The solid line shows susceptible (S), the dashed line illustrates infected (I) and the dashdot line represents AIDS cases (A) respectively

\section{Conclusion}

A sex-structured model for heterosexual transmission of HIV/AIDS in a community is developed in this paper. We use the model to assess the peer-education campaign as a single strategy approach in HIV prevention. We begin by analyzing the sex-structured model by determining its equilibrium, solvability, thresholds and stability. The model is extended to incorporate the effects of peer-education campaign (14). The basic reproduction number obtained (18) is a function of the parameters of peer-education campaign and when there is effective educational campaigns, the parameters $\pi_{i}=a_{i}=b_{i}=0$ and $\alpha_{i}>0$. For this case we shall have $\lim _{\pi_{i}, a_{i}, b_{i} \rightarrow 0} R_{0}=R_{1}=0$ and the effective peer-group educational campaigns in a community, the number of secondary infections is reduced to zero (figure 3 and 4 ) and the cases of new AIDS cases will also reduce to zero. Thus the effective educational campaigns help slow or eradicate the epidemic if properly implemented in communities. For Rwanda, to continue supporting Clubs against HIV and any other form of campaign that can help people to better know the methods of transmission of HIV 


\section{References}

[1] L. Cai, X. Li, M. Ghosh, B. Guo. Stability analysis of an HIV/AIDS epidemic model with treatment. J. Comput. Appl. Math 229 (2009) 313-323.

[2] Castilo-Chavez, C. W. Huang, J. Li, Competitive exclusion in gonorrhea models and other sexually- transmitted diseases, math.la.asu.edu/chavez/1996/JB77.pdf.

[3] Castilo-Chavez, C. F. Brauer, J.X. Velasco-Hernandez, Recruitment effects in heterosexually transmitted disease models, math.la,asu.edu/Chavez/1996/JB310.pdf.

[4] Diekmann, Heesterbeek, J. A. P, Metz On the definition and computation of the basic reproductive ratio $R_{0}$ in models for infectious diseases in heterogeneous populations, J. Mat. Bio. 28 (1990)365-382.

[5] Greenhalgh, D., Doyle, M. Lewis, F. A mathematical treatment of AIDS and condom use, IMA J. Math. Appl. Med. Biol. 18, No. 3, (2001) 225-249.

[6] Hsieh, Y. H. A two-sex model for treatment of HIV behavior change in a population of varying size, IMA J Math Appl. MED. Biol. 1996 Sep; 13(2001) 151-173.

[7] Hsieh, Y. H. Sheu, S.P., The effects of density-dependent treatment and behavior change on the dynamics of HIV transmission, J. Math. Biol. 43(2001)69-80.

[8] Hyman, J. M., Li, J., Stanley, E. A., The differential infectivity and staged progression models for the transmission of HIV, Mathematical Biosciences 155 (1999) 77-109.

[9] Hyman, J. M. J. Li, Stanley E.A., The reproductive number for an HIV model with differential infectivity and staged progression, Linear algebra and its application 398(2005) 101-116.

[10] Hyman, J. M., Stanley E. A., Using mathematical models to understand the AIDS epidemic, mathematical biosciences 90(1998) 415-473.

[11] Moghadas, S. M., Gumel, A. B., MeLeod R. G., Gordon R, could condoms stop the AIDS epidemic, www.umanitoba.ca/faculties/medecine/radiology/Dick-Gordonpapers/Moghadas\%20et\%20al.\%20(2004).pdf

[12] Mugisha, J.Y.T. The mathematical dynamics of HIV/AIDS epidemic in age-structured population, proceedings of Xi SISMA Symposium on the potential of mathematical modeling of problems from the SASMA region, Ed, Masanja VG, (1997)8-23.

[13] National institute of allergies and infections diseases diseases (NIAID). HIV infection in women [fact sheet]. Bethesda, Maryland: www.niaid.nih.gov/factsheets/womenhiv.htm.

[14] Reproductive health outlook (RHO), http//www/AIDS/HIV-AIDS special focuscondoms.html.

[15] UNAIDS report on the global AIDS epidemic 2012.

[16] United states department of health and human services, scientific Review panel confirms condoms are effective against HIV/AIDS, but epidemiological studies are insufficient for others STDs (press release) (2001), www.hhs.gov/news/pres/2001pres/20010720.html.

[17] Watmough J., Van den Driesche P., Reproduction number and sub-threshold endemic equilibria for compartmental models of diseases transmission, mathematical biosciences, 180(2002)29-48.

[18] WHO/UNAIDS. The male latex condom, condom programming fact sheets. Geneva: WHO (2001). 
[19] R. Zurakowski, A.R. Teel. A model predictive control based scheduling method for HIV therapy. Journal of Theoretical Biology 238 (2006) 368-382. 